

\title{
Backstepping with bounded feedbacks for systems not in feedback form
}

\author{
Frederic Mazenc, Abderrahman Iggidr
}

\section{To cite this version:}

Frederic Mazenc, Abderrahman Iggidr. Backstepping with bounded feedbacks for systems not in feedback form. 5th IFAC Symposium on Nonlinear Control Systems, Jul 2001, St Petersburg, Russia. pp.645-650. hal-01862865

\section{HAL Id: hal-01862865 \\ https://hal.inria.fr/hal-01862865}

Submitted on 29 Aug 2018

HAL is a multi-disciplinary open access archive for the deposit and dissemination of scientific research documents, whether they are published or not. The documents may come from teaching and research institutions in France or abroad, or from public or private research centers.
L'archive ouverte pluridisciplinaire HAL, est destinée au dépôt et à la diffusion de documents scientifiques de niveau recherche, publiés ou non, émanant des établissements d'enseignement et de recherche français ou étrangers, des laboratoires publics ou privés. 


\title{
BACKSTEPPING WITH BOUNDED FEEDBACKS FOR SYSTEMS NOT IN FEEDBACK FORM
}

\author{
F. Mazenc, A. Iggidr \\ INRIA Lorraine, Projet CONGE, \\ ISGMP Bât. A, Ile du Saulcy \\ 57045 Metz Cedex 01, France \\ email: mazenc@loria.fr,iggidr@loria.fr
}

\begin{abstract}
An extension of the backstepping approach is proposed. It allows to globally asymptotically stabilize by bounded feedbacks families of nonlinear control systems which are not in feedback form. Copyright ${ }^{\circ} 2001$ IFAC
\end{abstract}

Keywords: Nonlinear control, Lyapunov function, stabilization.

\section{INTRODUCTION}

One of the most popular nonlinear technique of control design is the backstepping approach. Many works (see for instance (Tsinias, 1997), (Freeman and Kokotovic, 1996)) and chapters of books (see for instance (Kristic et al., 1995) and (Khalil, 1996)) are devoted to this recursive Lyapunov design procedure. The multiple advantages offered by this approach are well-known. Observe in particular that this technique yields a wide family of globally asymptotically stabilizing control laws, allows to address robustness issues and to solve adaptive problems.

In a recent work (Freeman and Praly, 1998), R. Freeman and L. Praly, have shown that for some systems (an $n$-dimensional chain of integrators for instance), bounded stabilizing feedbacks can be constructed by applying a new version of this technique. This important result applies to the systems in feedback form:

$$
\left\{\begin{array}{l}
\dot{x}=f(x)+g(x) y \\
\dot{y}=u
\end{array}\right.
$$

where $u \in \mathbb{R}$ is the input, $x \in \mathbb{R}^{x}, y \in \mathbb{R}$ under suitable assumptions. The stabilizing control laws determined are simple and reminiscent of the nested saturation control laws used in (Teel, 1992a; Teel, 1992b) in another context.
In the present work, we complement (Freeman and Praly, 1998) in several directions:

- We consider systems which admit the following representation:

$$
\left\{\begin{array}{l}
\dot{x}=f(x)+g(x, y) y+h(x, y, u) u \\
\dot{y}=u
\end{array}\right.
$$

where $u \in \mathbb{R}$ is the input, $x \in \mathbb{R}^{x}, y \in \mathbb{R}$, which is larger than (1). Note in particular that due to the term $h(x, y, u) u$, the system (2) is not in feedback form. To the best of our knowledge, no backstepping result available in the literature applies to this system when $h(x, y, u) u$ is not smaller in norm than a function independent from $u$ or $h(0,0, u) u$ is not identically equal to zero. We do not impose these assumptions.

- We determine a family of stabilizing control laws, which contains elements arbitrarily small in norm and is different from the one proposed in (Freeman and Praly, 1998).

- The Lyapunov functions we construct are smooth and simpler than those proposed in (Freeman and Praly, 1998).

We will call our new method 'bounded backstepping approach'. However, it does not only complement the backstepping technique. It also sheds light on the forwarding procedure, which is a recursive Lyapunov design procedure exposed in (Teel, 1992b; Mazenc and Praly, 1996; Jankovic et 
al., 1996). On the one hand, from a practical point of view, our main result can be useful even when the forwarding approach can be theoretically applied to (2): this technique relies on change of coordinates (see (Mazenc and Praly, 1996)) or crossterms (see (Jankovic et al., 1996)) for which it is not always possible to determine explicit formulas when $f(x)$ is not linear and the bounded backstepping approach proceeds regardless of these tools. Moreover, the robustness properties which result from the forwarding approach are significantly different from those which result from our new extension of the backstepping approach. On the other hand, our approach applies to systems (2) which do not satisfy the following two standard assumptions of the forwarding:

(i) The system $\dot{x}=f(x)$ is globally stable

(ii) The function $\frac{|h(x, y, u)|}{1+|x|}$ is smaller than a function independent from $|x|$.

So far, the backstepping and the forwarding procedures were two separated techniques applicable respectively to lower-triangular and uppertriangular systems. Our work establishes a link between them: it shows that most of the feedforward systems can be stabilized by the bounded backstepping approach and gives tools enabling us to globally stabilize some systems which cannot be stabilized by combining the backstepping and the forwarding. These systems are termed noninterlaced systems (see (Sepulchre et al., 1996, Chapter 6) for the definition of interlaced systems) and, roughly speaking, are between the feedforward and the feedback systems.

The key feature of our work is a new choice of coordinates. It is very easy to understand why this crucial choice leads to bounded feedbacks with bounded derivatives along the trajectories. In our opinion, this simple change of coordinates is more important than the main result we give because this tool can be adapted to various problems, that, due to space limitation, we do not fully investigate.

\section{Preliminaries.}

1. The argument of the functions will be omitted whenever no confusion can arise from the context.

2. For a real valued $C^{1}$ function $k(\cdot)$, we denote by $k^{\prime}(\cdot)$ its first derivative.

3. We assume throughout the paper that the functions encountered are sufficiently smooth.

4. A positive definite function $V(\cdot)$ on $\mathbb{R}^{n}$ is a strict Lyapunov function for the system $\dot{\chi}=\varphi(\chi)$ if $\frac{\partial V}{\partial \chi}(\chi) \varphi(\chi)<0, \forall \chi \neq 0$.
5. A real-valued function $k(\cdot)$ is of class $\mathcal{K}_{\infty}$ if it is zero at zero, strictly increasing and goes to the infinity when its argument goes to the infinity.

6 . For a given $\varepsilon>0$, we denote by $\lambda(s)$ an even real-valued function such that

- $\lambda(s)=1$ when $s \in[0,3 \varepsilon]$.

- $\lambda(s)=0$ when $|s| \in[4 \varepsilon,+\infty[$.

- $\lambda(s) \in[0,1]$ for all $s$.

- $\left|\lambda^{\prime}(s)\right| \leq \frac{4}{\varepsilon},\left|\lambda^{\prime \prime}(s)\right| \leq \frac{16}{\varepsilon^{2}}$ for all $s$.

7. For a given $\left.\varepsilon \in] 0, \frac{1}{8}\right]$, we denote by $\sigma(s)$ an odd nondecreasing saturation such that

- $\sigma(s)=\varepsilon^{2} s$ for all $s \in[0, \varepsilon]$.

- $\sigma(s)=2 \varepsilon^{3}$ for all $s \in[3 \varepsilon,+\infty[$.

- $\left|\sigma^{\prime}(s)\right| \leq \varepsilon^{2},\left|\sigma^{\prime \prime}(s)\right| \leq \varepsilon$.

\section{INTRODUCTORY EXAMPLE}

Before stating and proving the main result of the work, we apply it to a simple example to show the key ideas of the approach. In particular, this benchmark example shows that the approach can be applied repeatedly.

Fact 2.1. The three-dimensional system

$$
\left\{\begin{array}{l}
\dot{x}_{1}=x_{2}+x_{3}^{2} \\
\dot{x}_{2}=x_{3} \\
\dot{x}_{3}=u
\end{array}\right.
$$

where $u$ is the input can be globally asymptotically stabilized and locally exponentially stabilized by state feedbacks arbitrarily small in norm using the bounded backstepping approach.

\section{Discussion:}

- The system (3) is a feedforward system and it can be globally asymptotically stabilized using the forwarding approach. Although our new strategy of design applies to some noninterlaced systems, we have chosen to introduce the main ideas of the work via the system (3) because the calculations involved in that case can be easily followed and therefore the various phenomena encountered can be easily understood.

- Due to the term $x_{3}^{2}$, the system (3) is not in feedback form and has not the structure of the systems studied in (Freeman and Praly, 1998). One cannot even apply to the reduced order system

$$
\left\{\begin{array}{l}
\dot{x}_{1}=x_{2}+\mu_{3}^{2} \\
\dot{x}_{2}=\mu_{3}
\end{array}\right.
$$

with $\mu_{3}$ as fictitious input, the results on backstepping available in the literature to prove its global asymptotic stabilizability. To understand why, let us try to apply the standard backstepping approach. We perform the change of coordinate 


$$
\xi_{2}=x_{2}-\mu_{2 s}\left(x_{1}\right)
$$

where $\mu_{2 s}\left(x_{1}\right)$ is any function which globally asymptotically stabilizes the system $\dot{x}_{1}=\mu_{2}$. It transforms (4) into

$$
\left\{\begin{array}{l}
\dot{x}_{1}=\mu_{2 s}\left(x_{1}\right)+\xi_{2}+\mu_{3}^{2} \\
\dot{\xi}_{2}=\mu_{3}-\mu_{2 s}^{\prime}\left(x_{1}\right)\left(x_{2}+\mu_{3}^{2}\right)
\end{array}\right.
$$

The next step of the backstepping approach consists in finding a function $\mu_{3}$ such that

$$
\mu_{3}-\mu_{2 s}^{\prime}\left(x_{1}\right)\left(x_{2}+\mu_{3}^{2}\right) \leq 0, \forall \xi_{2}>0, \forall x_{1}
$$

Since $\dot{x}_{1}=\mu_{2 s}\left(x_{1}\right)$ is globally asymptotically stable, there exists $x_{1 p}$ such that $-\mu_{2 s}^{\prime}\left(x_{1 p}\right)=$ $p>0$ and, on the other hand, there exists $M$ such that, for all $x_{2} \geq M$ then $x_{2}-\mu_{2 s}\left(x_{1 p}\right)>0$. It follows that if ( 7$)$ is satisfied, then

$$
\mu_{3}+p\left(x_{2}+\mu_{3}^{2}\right) \leq 0, \forall x_{2} \geq M, \forall x_{1}
$$

But when $x_{2} \geq M+1+\frac{1}{4 p^{2}}$, then

$$
\mu_{3}+p\left(x_{2}+\mu_{3}^{2}\right)>0
$$

This yields a contradiction.

We will show that the obstacle encountered can be overcome by selecting a very specific change of coordinates, which is the crucial tool introduced in our work. For example, for the system (4), instead of the variable $\xi_{2}$ defined in (5), we will select the variable

$$
X_{2}=x_{2}-\mu_{2 s}\left(x_{1}\right) \lambda\left(x_{2}\right)
$$

where $\mu_{2 s}\left(x_{1}\right)$ is a bounded function with a bounded first and second derivatives and $\lambda\left(x_{2}\right)$ the function introduced in the preliminaries.

- As already pointed out, we extensively utilize change of coordinates. When we write a system in new coordinates, we often keep some terms where the old coordinates are involved. This slight abuse of notation simplifies the analysis and the reading and does not prevent us from carrying out rigorous constructions.

Proof of Fact 2.1. Let $\varepsilon$ be a strictly positive real number smaller than $\frac{1}{40}$.

First Step. Stabilization of a first order system. The feedback

$$
\mu_{2}\left(x_{1}\right)=-\sigma\left(x_{1}\right)
$$

where $\sigma(\cdot)$ is the function defined in the preliminaries, globally asymptotically stabilizes the system

$$
\dot{x}_{1}=\mu_{2}
$$

Second Step. Stabilization of a second order system.

Consider the system (4). The change of coordinate

$$
X_{2}=x_{2}-\mu_{2}\left(x_{1}\right) \lambda\left(x_{2}\right)
$$

which is a global change of coordinate because $1-\mu_{2}\left(x_{1}\right) \lambda^{\prime}\left(x_{2}\right) \geq 1-8 \varepsilon^{2} \geq \frac{1}{2}$ transforms the system (3) into

$$
\left\{\begin{aligned}
\dot{x}_{1}= & \mu_{2}\left(x_{1}\right) \lambda\left(x_{2}\right)+X_{2}+\mu_{3}^{2} \\
\dot{X}_{2}= & {\left[1-\mu_{2}\left(x_{1}\right) \lambda^{\prime}\left(x_{2}\right)\right] \mu_{3}-} \\
& \mu_{2}^{\prime}\left(x_{1}\right)\left(x_{2}+\mu_{3}^{2}\right) \lambda\left(x_{2}\right)
\end{aligned}\right.
$$

The equation of second order

$$
\begin{gathered}
{\left[1-\mu_{2}\left(x_{1}\right) \lambda^{\prime}\left(x_{2}\right)\right] \mu_{3}-\mu_{2}^{\prime}\left(x_{1}\right)\left(x_{2}+\mu_{3}^{2}\right) \lambda\left(x_{2}\right)=} \\
-\sigma\left(X_{2}\right)
\end{gathered}
$$

admits a solution if its discriminant $\Delta$ is nonnegative.

Using the definition of $\mu_{2}\left(x_{1}\right)$ and the properties satisfied by $\sigma(\cdot)$ and $\lambda(\cdot)$, we deduce

$$
\begin{aligned}
\Delta= & {\left[1+\sigma\left(x_{1}\right) \lambda^{\prime}\left(x_{2}\right)\right]^{2} } \\
& -4\left[\sigma^{\prime}\left(x_{1}\right)^{2} \lambda\left(x_{2}\right)^{2} x_{2}+\sigma^{\prime}\left(x_{1}\right) \lambda\left(x_{2}\right) \sigma\left(X_{2}\right)\right] \\
\geq & \frac{39^{2}}{40^{2}}-24 \varepsilon^{5}>\frac{1}{2} .
\end{aligned}
$$

It follows readily that the feedback

$$
\mu_{3}\left(x_{1}, x_{2}\right)=-\frac{2\left[\sigma^{\prime}\left(x_{1}\right) \lambda\left(x_{2}\right) x_{2}+\sigma\left(X_{2}\right)\right]}{1+\sigma\left(x_{1}\right) \lambda^{\prime}\left(x_{2}\right)+\sqrt{\Delta}}
$$

is well-defined, zero at the origin, smooth and solution of the above second oredr equation. Moreover, $\mu_{3}\left(x_{1}, x_{2}\right)$ is smaller in norm than $24 \varepsilon^{3}$. The system (13) in closed-loop with this feedback is

$$
\left\{\begin{array}{l}
\dot{x}_{1}=-\sigma\left(x_{1}\right) \lambda\left(x_{2}\right)+X_{2}+\mu_{3}\left(x_{1}, x_{2}\right)^{2} \\
\dot{X}_{2}=-\sigma\left(X_{2}\right)
\end{array}\right.
$$

We prove the global asymptotic stability of this system by a Lyapunov approach. Consider the positive definite and radially unbounded function $^{1}$

$$
V\left(x_{1}, x_{2}\right)=\int_{0}^{x_{1}} \sigma(s) d s+k\left(X_{2}^{2}\right)
$$

where $k(\cdot)$ is a smooth function of class $\mathcal{K}_{\infty}$ with a strictly positive first derivative. Its derivative along the trajectories of (14) satisfies:

$$
\begin{aligned}
& \dot{V}\left(x_{1}, x_{2}\right)=-\sigma\left(x_{1}\right)^{2} \lambda\left(x_{2}\right) \\
& \quad+\sigma\left(x_{1}\right)\left[X_{2}+\mu_{3}\left(x_{1}, x_{2}\right)^{2}\right]-2 k^{\prime}\left(X_{2}^{2}\right) X_{2} \sigma\left(X_{2}\right) \\
& =-\sigma\left(x_{1}\right)^{2}+\sigma\left(x_{1}\right)^{2}\left[1-\lambda\left(x_{2}\right)\right]+\sigma\left(x_{1}\right) X_{2} \\
& \quad+\sigma\left(x_{1}\right) \mu_{3}\left(x_{1}, x_{2}\right)^{2}-2 k^{\prime}\left(X_{2}^{2}\right) X_{2} \sigma\left(X_{2}\right)
\end{aligned}
$$

Using the properties satisfied by $\sigma(\cdot)$ and $\lambda(\cdot)$, we deduce that one can determine positive real numbers $C_{1}, C_{2}, C_{3}$ such that

$$
\begin{array}{r}
\left|\sigma\left(x_{1}\right)^{2}\left[1-\lambda\left(x_{2}\right)\right]\right| \leq C_{1} X_{2} \sigma\left(X_{2}\right) \\
\left|\sigma\left(x_{1}\right) X_{2}\right| \leq \frac{1}{4} \sigma\left(x_{1}\right)^{2}+C_{2} X_{2} \sigma\left(X_{2}\right) \\
\left|\sigma\left(x_{1}\right) \mu_{3}\left(x_{1}, x_{2}\right)^{2}\right| \leq \varepsilon^{2} \sigma\left(x_{1}\right)^{2}+C_{3} X_{2} \sigma\left(X_{2}\right)
\end{array}
$$

From (16),(18),(17), we deduce that

$$
\begin{aligned}
\dot{V}\left(x_{1}, x_{2}\right) \leq & \left(-\frac{3}{4}+5 \varepsilon^{2}\right) \sigma\left(x_{1}\right)^{2} \\
& +\left(C_{1}+C_{2}+C_{3}\right) X_{2} \sigma\left(X_{2}\right) \\
& -2 k^{\prime}\left(X_{2}^{2}\right) X_{2} \sigma\left(X_{2}\right)
\end{aligned}
$$

\footnotetext{
1 The function $V(\cdot)$ is radially unbounded because $\sigma(\cdot)$ is not decreasing.
} 
Choosing $k(s)=(K+1) s$ with $K=C_{1}+C_{2}+C_{3}$, we obtain

$$
\begin{aligned}
\dot{V}\left(x_{1}, x_{2}\right) & \leq-\frac{1}{2} \sigma\left(x_{1}\right)^{2}-(K+1) X_{2} \sigma\left(X_{2}\right) \\
& <0, \forall\left(x_{1}, x_{2}\right) \neq(0,0) .
\end{aligned}
$$

Third Step. Stabilization of the system (3).

Using the coordinate $X_{2}$ defined in (12) and the coordinate

$$
X_{3}=x_{3}-\mu_{3}\left(x_{1}, x_{2}\right) \lambda\left(x_{3}\right)
$$

the system (3) becomes

$$
\left\{\begin{aligned}
\dot{x}_{1}= & \mu_{2}\left(x_{1}\right) \lambda\left(x_{2}\right)+X_{2}+x_{3}^{2} \\
\dot{X}_{2}= & {\left[1-\mu_{2}\left(x_{1}\right) \lambda^{\prime}\left(x_{2}\right)\right] x_{3} } \\
& -\mu_{2}^{\prime}\left(x_{1}\right)\left(x_{2}+x_{3}^{2}\right) \lambda\left(x_{2}\right) \\
\dot{X}_{3}= & {\left[1-\mu_{3}\left(x_{1}, x_{2}\right) \lambda^{\prime}\left(x_{3}\right)\right] u } \\
& +\left[\frac{\partial \mu_{3}}{\partial x_{1}}\left(x_{2}+x_{2}^{2}\right)+\frac{\partial \mu_{3}}{\partial x_{2}} x_{3}\right] \lambda\left(x_{3}\right)
\end{aligned}\right.
$$

One can check readily that $\frac{\partial \mu_{3}}{\partial x_{1}}\left(x_{1}, x_{2}\right)\left(x_{2}+x_{2}^{2}\right)$ and $\frac{\partial \mu_{3}}{\partial x_{2}}\left(x_{1}, x_{2}\right) x_{3} \lambda\left(x_{3}\right)$ are bounded in norm and $\left[1-\mu_{3}\left(x_{1}, x_{2}\right) \lambda^{\prime}\left(x_{3}\right)\right] \geq \frac{1}{2}$. It follows that the feedback

$$
\begin{gathered}
u\left(x_{1}, x_{2}, x_{3}\right)= \\
-\frac{\left[\frac{\partial \mu_{3}}{\partial x_{1}}\left(x_{1}, x_{2}\right)\left(x_{2}+x_{2}^{2}\right)+\frac{\partial \mu_{3}}{\partial x_{2}}\left(x_{1}, x_{2}\right) x_{3}\right] \lambda\left(x_{3}\right)}{1-\mu_{3}\left(x_{1}, x_{2}\right) \lambda^{\prime}\left(x_{3}\right)} \\
-\frac{\sigma\left(X_{3}\right)}{1-\mu_{3}\left(x_{1}, x_{2}\right) \lambda^{\prime}\left(x_{3}\right)}
\end{gathered}
$$

is well-defined and bounded in norm. The system (20) in closed-loop with this feedback is

$$
\left\{\begin{aligned}
\dot{x}_{1}= & \mu_{2}\left(x_{1}\right) \lambda\left(x_{2}\right)+X_{2}+x_{3}^{2} \\
\dot{X}_{2}= & {\left[1-\mu_{2}\left(x_{1}\right) \lambda^{\prime}\left(x_{2}\right)\right] x_{3} } \\
& -\mu_{2}^{\prime}\left(x_{1}\right)\left(x_{2}+x_{3}^{2}\right) \lambda\left(x_{2}\right) \\
\dot{X}_{3}= & -\sigma\left(X_{3}\right)
\end{aligned}\right.
$$

Using (19), we straightforwardly deduce that the inequality

$$
\begin{aligned}
\dot{V} \leq & -\frac{1}{2} \sigma\left(x_{1}\right)^{2}-(K+1) X_{2} \sigma\left(X_{2}\right) \\
& +\sigma\left(x_{1}\right)\left(x_{3}^{2}-\mu_{3}\left(x_{1}, x_{2}\right)^{2}\right) \\
& +2(K+1)\left|X_{2}\right| R\left(x_{1}, x_{2}, x_{3}\right)
\end{aligned}
$$

with

$$
\begin{aligned}
R\left(x_{1}, x_{2}, x_{3}\right)= & \left|\left[1+\sigma\left(x_{1}\right) \lambda^{\prime}\left(x_{2}\right)\right]\left[x_{3}-\mu_{3}\left(x_{1}, x_{2}\right)\right]\right| \\
& +\left|\sigma^{\prime}\left(x_{1}\right)\left[x_{3}^{2}-\mu_{3}\left(x_{1}, x_{2}\right)^{2}\right] \lambda\left(x_{2}\right)\right|
\end{aligned}
$$

is satisfied. One can determine a strictly positive real number $C_{4}$ such that

$$
\left|x_{3}-\mu_{3}\left(x_{1}, x_{2}\right)\right| \leq C_{4}\left|X_{3}\right| .
$$

Using this inequality, one can prove that

$R\left(x_{1}, x_{2}, x_{3}\right) \leq C_{4}\left[\varepsilon\left|X_{3}\right|+2 \varepsilon\left|\mu_{3}\left(x_{1}, x_{2}\right)\right|+2\right]\left|X_{3}\right|$. and

$$
\begin{aligned}
& \left|\sigma\left(x_{1}\right)\left(x_{3}^{2}-\mu_{3}\left(x_{1}, x_{2}\right)^{2}\right)\right| \\
& \quad \leq C_{4}\left|\sigma\left(x_{1}\right)\right|\left|X_{3}\right|\left[\left|X_{3}\right|+2\left|\mu_{3}\left(x_{1}, x_{2}\right)\right|\right] .
\end{aligned}
$$

which allows us to write

$$
\begin{aligned}
\dot{V} \leq & -\frac{1}{2} \sigma\left(x_{1}\right)^{2}-(K+1) X_{2} \sigma\left(X_{2}\right) \\
& +\stackrel{C}{C}_{5}\left(\left|\sigma\left(x_{1}\right)\right|+\left|X_{2}\right|\right)\left[X_{3}^{2}+\left|X_{3}\right|\right]
\end{aligned}
$$

where $C_{5}$ is a strictly positive real number. This inequality and the properties of $X_{3}$-subsystem of (21) allow us to conclude immediately that the system is globally asymptotically stable and locally exponentially stable. Due to space limitation, we do not construct a Lyapunov function for the overall system but this construction presents no difficulty.

\section{MAIN RESULT}

In this section, we state and prove a general result based on the key ideas used in the previous section. In our opinion, these key ideas are more useful than the general result itself. For many systems which do not satisfy all the assumptions of our theorem can be stabilized by adapting the methodology we propose to their characteristics. Introduce a set of assumptions.

Assumption A1. For all $\varepsilon \in] 0,1]$, there exists a function $y^{*}(x)$ bounded in norm by $\varepsilon^{2}$, a positive definite radially unbounded function $V(x)$, a positive definite function $W(x)$ such that:

A11. The inequalities

$$
\frac{\partial V}{\partial x}(x)\left[f(x)+g\left(x, y^{*}(x)\right) y^{*}(x)\right] \leq-W(x)
$$

$\sup _{|y| \leq 2 \varepsilon}\left|\frac{\partial y^{*}}{\partial x}(x)\left(f(x)+g(x, y) y^{*}(x)\right)\right|^{2} \leq \varepsilon C W(x)(23)$

where $C$ is a positive constant independent of $\varepsilon$

$$
\begin{gathered}
\sup _{|u| \leq 10 \varepsilon}\left|\frac{\partial V}{\partial x}(x)[f(x)+g(x, y) y+h(x, y, u) u]\right| \\
\leq \kappa\left(\left|y-y^{*}(x)\right|\right)
\end{gathered}
$$

where $\kappa(\cdot)$ is a strictly positive function, are satisfied.

A12. The inequalities

$$
\begin{gathered}
\left|\frac{\partial y^{*}}{\partial x}(x) f(x)\right| \leq \frac{1}{2} \varepsilon, \sup _{|y| \leq 4 \varepsilon}\left|\frac{\partial y^{*}}{\partial x}(x) g(x, y)\right| \leq \frac{1}{8} \\
\left|\frac{\partial y^{*}}{\partial x}(x)\right| \sup _{|y| \leq 4 \varepsilon,|u| \leq 10 \varepsilon}\left|\frac{\partial h}{\partial u}(x, y, u)\right| \leq 1 \\
\left|\frac{\partial y^{*}}{\partial x}(x)\right| \sup _{|y| \leq 4 \varepsilon}|h(x, y, 0)| \leq \frac{1}{4}
\end{gathered}
$$


hold.

Assumption A2. For all $u$ smaller in norm than $10 \varepsilon$, the following inequality holds:

$$
\begin{aligned}
\left|\frac{\partial V}{\partial x}(x) \Gamma(x, y, u)\right| & \leq \frac{1}{4} W(x) \\
& +\rho\left(\left|y-y^{*}(x)\right|\right)\left|y-y^{*}(x)\right|^{2}+H|u|^{2}
\end{aligned}
$$

with $\Gamma(x, y, u)=g(x, y) y-g\left(x, y^{*}(x)\right) y^{*}(x)+$ $h(x, y, u) u$ and where $\rho(\cdot)$ is a positive function and $H$ a positive real number independent of $\varepsilon^{2}$.

Theorem 3.1. Assume Assumptions A1 to A2 are satisfied by the system (2). Then, for all $\varepsilon \in$ ]0,1], the system (2) is globally asymptotically stabilizable by feedback bounded in norm by $10 \varepsilon$.

Remark 1. When $V(x)$ and $W(x)$ are lower bounded on a neighborhood of the origin by a quadratic function, then the feedback constructed in the forthcoming proof globally asymptotically and locally exponentially stabilizes the system (2). Remark 2. When the function $h(x, y, u)$ is not affine in $u$ we prove the existence of a stabilizing control law, which is solution of a nonlinear equation, by invoking the fixed point theorem. So a priori, in that case, no explicit formula of stabilizing feedback can be determined. Fortunately, it is always possible to find approximating expressions of the solution of the nonlinear equation which are sufficiently accurate to be stabilizing.

Proof. Impose a priori on $\varepsilon$ to belong to the interval $\left.] 0, \frac{1}{40}\right]$. Then $1-y^{*}(x) \lambda^{\prime}(y) \geq \frac{1}{2}$, which implies that the variable

$$
Y=y-y^{*}(x) \lambda(y)
$$

is globally defined i.e. the function $(x, y) \rightarrow(x, y-$ $\left.y^{*}(x) \lambda(y)\right)$ is a global diffeomorphism.

The derivative of $Y$ along the trajectories of (2) is

$$
\begin{aligned}
\dot{Y}= & {\left[1-y^{*}(x) \lambda^{\prime}(y)\right] u } \\
& -\frac{\partial y^{*}}{\partial x}(x)(f(x)+g(x, y) y+h(x, y, u) u) \lambda(y) \\
= & {\left[1-y^{*}(x) \lambda^{\prime}(y)-\frac{\partial y^{*}}{\partial x}(x) h(x, y, 0) \lambda(y)\right] u } \\
& -\frac{\partial y^{*}}{\partial x_{x}}(x)[h(x, y, u)-h(x, y, 0)] u \lambda(y) \\
& -\frac{\partial y^{*}}{\partial x}(x)(f(x)+g(x, y) y) \lambda(y)
\end{aligned}
$$

According to the definition of $\lambda(\cdot)$, the fact that $\left|y^{*}(x)\right| \leq \varepsilon^{2}$ and inequality (27) in Assumption A12, it follows that

$$
\left|y^{*}(x) \lambda^{\prime}(y)+\frac{\partial y^{*}}{\partial x}(x) h(x, y, 0) \lambda(y)\right| \leq \frac{1}{2}
$$

Using the fixed point theorem, one can easily deduce from the inequalities (25) and (26) in

\footnotetext{
2 Since $\varepsilon$ belongs to the interval ]0,1], imposing on $H$ to be independent of $\varepsilon$ is not a restrictive requirement.
}

Assumption A12 that, when $\varepsilon$ is sufficiently small, there exists $u_{s}=u_{s}(x, y)$, smaller in norm than $10 \varepsilon$ such that ${ }^{3}$

$$
\begin{aligned}
& {\left[1-y^{*}(x) \lambda^{\prime}(y)-\frac{\partial y^{*}}{\partial x}(x) h(x, y, 0) \lambda(y)\right] u_{s}} \\
& -\frac{\partial y^{*}}{\partial x_{*}}(x)\left[h\left(x, y, u_{s}\right)-h(x, y, 0)\right] u_{s} \lambda(y) \\
& -\frac{\partial y^{*}}{\partial x}(x)(f(x)+g(x, y) y) \lambda(y)=-\sigma(Y)
\end{aligned}
$$

where $\sigma(Y)$ is the function defined in the preliminaries. When such a feedback is chosen, we have

$$
\dot{Y}=-\sigma(Y)
$$

The next objective is the construction of a strict Lyapunov function for the system (2) in closedloop with $u_{s}(x, y)$ using the functions $V(x)$ and $\frac{1}{2} Y^{2}$. The derivative of the

$$
U_{k}(x, y)=V(x)+k\left(\frac{1}{2} Y^{2}\right)
$$

where $k(\cdot)$ is a function of class $\mathcal{K}^{\infty}$ with a strictly positive first derivative, satisfies

$$
\dot{U}_{k}=\dot{V}-k^{\prime}\left(\frac{1}{2} Y^{2}\right) Y \sigma(Y)
$$

We distinguish between two cases.

First case: $|Y| \geq \varepsilon$. Then, according to inequality (24) in Assumption A11,

$$
\begin{aligned}
\dot{V} & \leq\left|\frac{\partial V}{\partial x}(x)\left[f(x)+g(x, y) y+h\left(x, y, u_{s}\right) u_{s}\right]\right| \\
& \leq \kappa(|Y|) \leq \frac{1}{2} k^{\prime}\left(\frac{1}{2} Y^{2}\right) Y \sigma(Y)
\end{aligned}
$$

when $k(\cdot)$ is chosen such that $k^{\prime}(\cdot)$ is sufficiently large ${ }^{4}$.

Second case: $|Y| \leq \varepsilon$. In this case $|y| \leq 2 \varepsilon$ which implies that $Y=y-y^{*}(x)$. The derivative of $V(\cdot)$ along the solutions of system (2) in closed-loop with $u_{s}=u_{s}(x, y)$ can be written:

$$
\begin{aligned}
\dot{V} & =\frac{\partial V}{\partial x}(x)\left[f(x)+g(x, y) y+h\left(x, y, u_{s}\right) u_{s}\right] \\
& \leq-W(x)+\frac{\partial V}{\partial x}(x) \Gamma\left(x, y, u_{s}(x, y)\right) .
\end{aligned}
$$

where $\Gamma(x, y, u)$ is the function defined in Assumption A2. According to Assumption A2, we deduce

$$
\dot{V} \leq-\frac{3}{4} W(x)+\rho(|Y|)|Y|^{2}+H\left|u_{s}(x, y)\right|^{2}
$$

Since $u_{s}(x, y)$ is solution of (29), we have, when $|Y| \leq \varepsilon\left(|y| \leq 2 \varepsilon, \lambda(y)=1, \lambda^{\prime}(y)=0\right)$,

\footnotetext{
3 When $h(x, y, u)$ is linear in $u$ an explicit expression can be determined simply by solving an equation of degree two. 4 The existence of such a function $k(\cdot)$ is almost obvious. Moreover, observe that from a practical point of view the determination of an explicit expression for $k(\cdot)$ is an easy task.
} 


$$
\begin{gathered}
u_{s}(x, y)= \\
\frac{\frac{\partial y^{*}}{\partial x} \tilde{h} u_{s}(x, y)+\frac{\partial y^{*}}{\partial x}[f(x)+g(x, y) y]-\sigma(Y)}{1-\frac{\partial y^{*}}{\partial x} h(x, y, 0)}
\end{gathered}
$$

where $\tilde{h}=\left[h\left(x, y, u_{s}(x, y)\right)-h(x, y, 0)\right]$.

Using the fact that $u_{s}(x, y)$ is bounded by $10 \varepsilon$, (27) and (26) imply $\left|\frac{\partial y^{*}}{\partial x} \tilde{h}\right| \leq\left|u_{s}(x, y)\right| \leq 10 \varepsilon$ and $\left|1-\frac{\partial y^{*}}{\partial x} h(x, y, 0)\right| \geq \frac{3}{4}$. Therefore, by writing $g(x, y) y=g(x, y) y^{*}(x)+g(x, y) Y$, we get

$$
\begin{aligned}
{\left[\frac{3}{4}-10 \varepsilon\right]\left|u_{s}\right| \leq } & \left|\frac{\partial y^{*}}{\partial x}(x)\left(f(x)+g(x, y) y^{*}(x)\right)\right| \\
& +\left|\frac{\partial y^{*}}{\partial x}(x) g(x, y) Y\right|+|\sigma(Y)|
\end{aligned}
$$

It follows from this inequality and (23) in A11 and (25) in A12 that one can determine a positive real number $P$, independent of $\varepsilon$, such that

$$
H u_{s}(x, y)^{2} \leq \varepsilon P W(x)+P|Y|^{2}
$$

Choosing $\varepsilon$ such that $\varepsilon P \leq \frac{1}{4}$ and denoting $\tilde{\rho}(|Y|)=\rho(|Y|)+P$, we deduce from (35) and (34) that

$$
\begin{aligned}
\dot{V} & \leq-\frac{1}{2} W(x)+\tilde{\rho}(|Y|)|Y|^{2} \\
& \leq-\frac{1}{2} W(x)+\frac{1}{2} k^{\prime}\left(\frac{1}{2} Y^{2}\right) Y \sigma(Y)
\end{aligned}
$$

when $k(\cdot)$ is chosen such that $k^{\prime}(\cdot)$ is sufficiently large ${ }^{5}$.

Finally, thanks to (33) and (36), one can conclude that

$$
\dot{U}_{k} \leq-\frac{1}{2} k^{\prime}\left(\frac{1}{2} Y^{2}\right) Y \sigma(Y)
$$

when $|Y|>\varepsilon$ and

$$
\dot{U}_{k} \leq-\frac{1}{2} W(x)-\frac{1}{2} k^{\prime}\left(\frac{1}{2} Y^{2}\right) Y \sigma(Y)
$$

when $|Y|<\varepsilon$. One can check readily that the derivative of $U_{k}(\cdot)$ along the trajectories of the system is definite negative.

This concludes the proof.

\section{CONCLUDING REMARKS}

We have presented an extension of the backstepping approach which yields bounded stabilizing feedbacks and strict Lyapunov functions. The key feature of this strategy of design is the introduction of a particular change of coordinate. This key idea can be used to solve various open stabilization problems. A discrete-time version and a timevarying of Theorem 3.1 can be proved. In particular, by adapting our method, we conjecture that one can solve some open problems of tracking of trajectories of nonholonomic systems by bounded feedback. It can also be used to exhibit a family of globally asymptotically stabilizable noninterlaced systems: observe that it allowed us in particular to construct a strict Lyapunov function for the 'ball and beam' system (see (Mazenc et al., 1998)). Further studies will be devoted to these problems.

\section{REFERENCES}

Freeman, R. and P. Kokotovic (1996). Robust Nonlinear Control Design. Birkhauser, Boston.

Freeman, R. and L. Praly (1998). Integrators Backstepping for Bounded Controls and Control Rates. IEEE Trans. on Autom. Control, 43, 258-262.

Jankovic, M.J., R. Sepulchre and P.V. Kokotovic (1996). Global stabilization of an enlarged class of cascade nonlinear systems. IEEE Trans. on Autom. Control, 41, no.12, 1723-1735.

Khalil, H. (1996). Nonlinear Systems. 2nd ed. Prentice Hall.

Kristic, M., I. Kanellakopoulos and P. Kokotovic (1995). Nonlinear and Adaptive Control Design. Wiley-Interscience, New York.

Mazenc, F., A. Astolfi and R. Lozano (1998). Lyapunov Function for the Ball and Beam: Robustness Property. 37th IEEE Conference on decision and control.

Mazenc, F. and L. Praly (1996). Adding an integration and Global asymptotic stabilization of feedforward systems. IEEE Trans. on $A u$ tom. Control, 41, no.11, 1559-1578.

Sepulchre, R., M.J. Jankovic and P.V. Kokotovic (1996). Constructive Nonlinear Control. Springer-Verlag.

Teel, A. (1992a). Using saturation to stabilize a class of single-input partially linear composite systems. Proc. IFAC Nonlinear Contr. Syst. Design Symp., Bordeau, France, June 1992.

Teel, A. (1992b). Feedback stabilization: nonlinear solutions to inherently nonlinear problems. Univ. California, Berkeley, CA. Tech Rep. UCB/ERLM92/65, June 1992.

Tsinias, J. (1997). Input to State Stability Properties of Nonlinear Systems and Applications to Bounded Feedback Stabilization Using Saturation. ESAIM: Control, Optimization and Calculus of Variations., 2, 57-85. 